\title{
Status and Prospects of the Auger Engineering Radio Array
}

\author{
Johannes Schulz $^{* a}$ for the Pierre Auger Collaboration ${ }^{b}$ \\ ${ }^{a}$ Department of Astrophysics/IMAPP, Radboud University, Nijmegen, The Netherlands \\ ${ }^{b}$ Observatorio Pierre Auger, Av. San Martín Norte 304, 5613 Malargüe, Argentina \\ E-mail: auger_spokespersons@fnal.gov \\ Full author list: http://www.auger.org/archive/authors_2015_06.html
}

The Auger Engineering Radio Array (AERA) is an extension of the Pierre Auger Observatory. It is used to detect radio emission from extensive air showers in the $30-80 \mathrm{MHz}$ frequency band. A focus of interest is the dependence of the radio emission on shower parameters such as the energy and the atmospheric depth of the shower maximum. After three phases of deployment, AERA now consists of 153 autonomous radio stations with different spacings, covering an area of about $17 \mathrm{~km}^{2}$. The size, station spacings, and geographic location at the same site or near other Auger extensions, are all targeted at cosmic ray energies above $10^{17} \mathrm{eV}$. The array allows us to explore different technical schemes to measure the radio emission as well as to cross calibrate our measurements with the established baseline detectors of the Auger Observatory. We present the most recent technological developments and selected experimental results obtained with AERA.

The 34th International Cosmic Ray Conference,

30 July - 6 August, 2015

The Hague, The Netherlands

\footnotetext{
*Speaker.
} 


\section{Introduction}

The subject of radio detection of extensive air showers has developed rapidly over the last years [1]. The performance, in terms of energy and composition measurements, is quickly approaching a competitive level compared to the air-fluorescence technique [2, 3]. In terms of duty cycle, however, the radio technique is advantageous as the operation is only limited in case of strong atmospheric electric fields (e.g., during thunderstorms) which significantly affect the radio emission processes [4]. Therefore a duty cycle close to $100 \%$ can be obtained which is approximately seven times higher than that for air-fluorescence measurements that can only take place during clear moonless nights.

By acquiring detailed information about the polarization of the radio pulses [5], an understanding of the two fundamental emission mechanisms, geomagnetic and charge excess emission, has been established. Together with the convergence between predictions of Monte Carlo simulation codes and the radio measurements [3], significant improvements were achieved in the parametrization of the lateral energy density distribution function $[6,7]$. The shape of the radio wave front has been found to be hyperbolic $[8,9]$ and the shape of the measured pulses is also the subject of intense research [10]. With these developments, parameters which are sensitive to the air shower development (often characterized by the atmospheric depth of maximum shower development: $\mathrm{X}_{\max }$ ) and to the properties of the initial cosmic ray have been identified. Their cross calibrations with established detection techniques can only be performed with hybrid detector systems.

The Auger Engineering Radio Array is dedicated to multi-hybrid detections of cosmic rays at the Pierre Auger Observatory [11]. It is conveniently co-located within the low-energy enhancement AMIGA and the high elevation fluorescence telescopes (HEAT). AMIGA is an infill region of the surface detector array (SD) with a spacing of $750 \mathrm{~m}$ and six associated muon counters. HEAT is an extension of the fluorescence detector (FD) which has an elevated field of view. All together, four different detection techniques are used to detect the same showers which allows us to perform multi detector cross calibrations and analyses. In addition, the systems can trigger each other. This constellation provides unique and optimal conditions for cross calibrations, further technical developments and pathfinder studies for future large scale experiments.

\section{The Auger Engineering Radio Array}

The AERA project has been carried out in three phases. AERA24, an array of 24 radio detection stations (RDS) was deployed in September 2010 to prove the feasibility of radio+particle hybrid air shower measurements, to develop the techniques to reconstruct air shower parameters from the radio data and to investigate the radio emission mechanisms [2, 5]. In a second stage, leading to AERA124, 100 RDS were deployed with a modified design in May 2013. With different grid sizes (144 m, $250 \mathrm{~m}, 375 \mathrm{~m}$ ), multiple improved hardware and trigger concepts and an instrumented area of $6 \mathrm{~km}^{2}$, AERA124 detected several thousand events per year. In the third stage, AERA153 covers about $17 \mathrm{~km}^{2}$ since April 2015, with $375 \mathrm{~m}$ and $750 \mathrm{~m}$ spacing between the additional RDS. The targets of this extension are mainly horizontal showers $\left(>60^{\circ}\right.$ zenith angle) with large scale radio footprints. The layout of AERA including all three stages is shown in figure 1 together with the other co-located detector systems.

The AERA24 stations are equipped with two logarithmic-periodic dipole antennas (LPDA) [12], oriented in magnetic North-South and East-West directions. The signals are amplified and filtered 


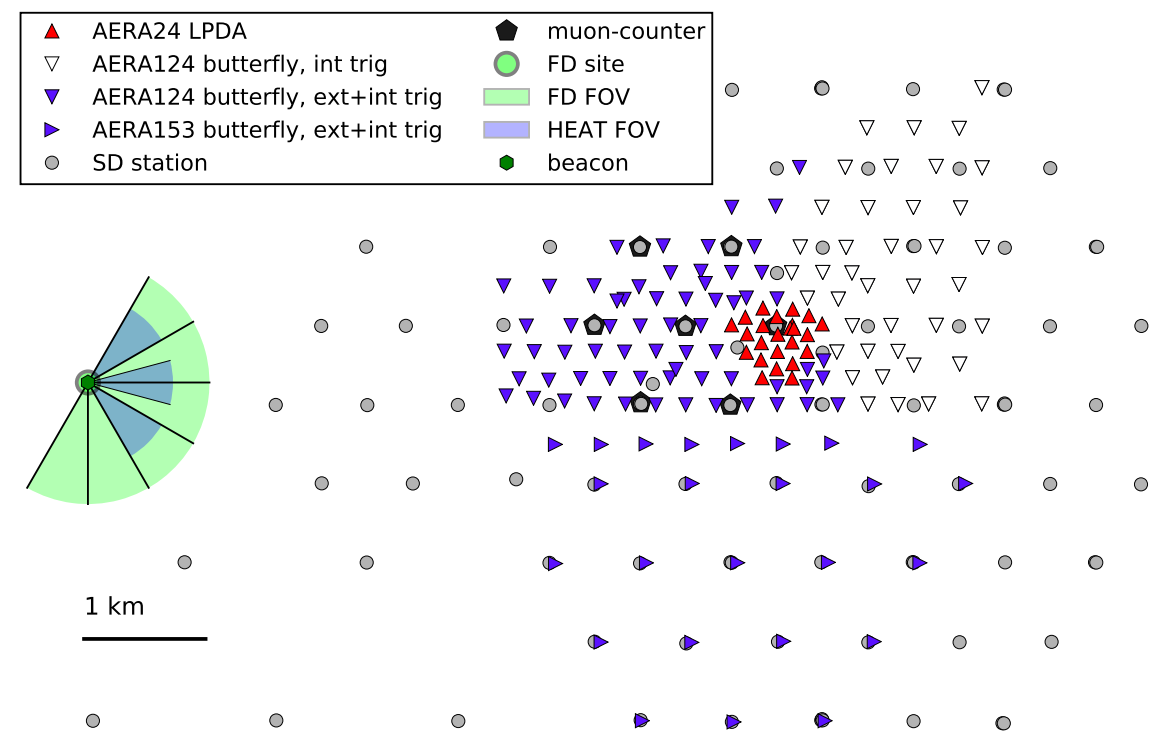

Figure 1: Schematic overview of the Auger Engineering Radio Array and part of the surface detector array and AMIGA. One site of the fluorescence detector and the HEAT extension are also indicated with their field of view. The reference beacon for timing calibration is located at the FD site.

before they are introduced into a filter-amplifier and digitization chain. There, they are band pass filtered between $30-80 \mathrm{MHz}$, digitized and further processed by a field programmable gate array (FPGA) and a central processing unit (CPU). This chain exists in various forms to test and optimize the technical realization of different detection strategies. For AERA24, two versions were deployed focusing on different triggering methods. One has a sampling rate of $180 \mathrm{MHz}$ and features a $4 \mathrm{~GB}$ ring buffer which allows for external triggering by other detectors of the Pierre Auger Observatory. The other has a sampling rate of $200 \mathrm{MHz}$ and is designed for self-triggering. In case of self-triggering, a first trigger decision can be made in the FPGA. This trigger information is then forwarded to the CPU where further processing and signal verification are done. Remaining triggers are GPS time stamped and sent to the central radio data acquisition system where all time stamps of triggered stations are processed. In case of coincidences between RDS, the data are read out, formed into an event and saved to disk. Data from externally triggered stations are directly saved to disk for offline analyses. The communication and data transfer of AERA24 is handled via optical fiber connections. The antenna type used for the new stations of AERA124 and AERA153 is an active bowtie antenna also called "butterfly" $[13,12]$. This antenna is highly sensitive towards the ground which enhances the antenna gain. The general layout of the digitizing chain is similar to the one used in AERA24. In total, 89 butterfly RDS are equipped with deeply buffering hardware and 40 butterfly RDS are employing internal triggering only. This internal trigger is based on radio self-triggering and on small scintillation counters in the electronics compartment of the radio station itself. The 40 internally triggered stations (see fig. 1) are currently not included in the data set presented here. Due to the increased size of the array, wireless links were introduced for the new stations. All 153 stations operate autonomously employing solar power systems.

The AERA data are merged with data from the other detector systems and subsequently analyzed with the software package Offline including the dedicated extension for radio [14]. During the event 


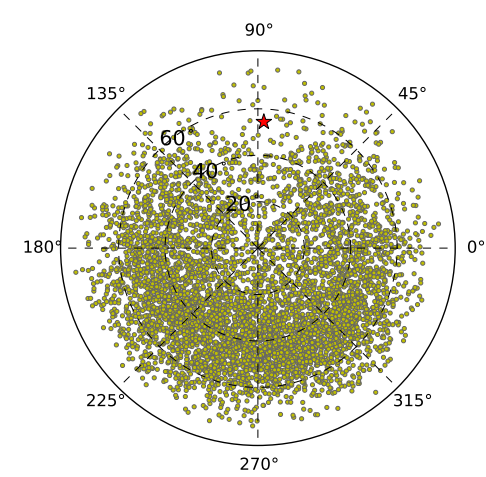

Figure 2: Skyplot of AERA+SD hybrid data. The star indicates the direction of the geomagnetic field.

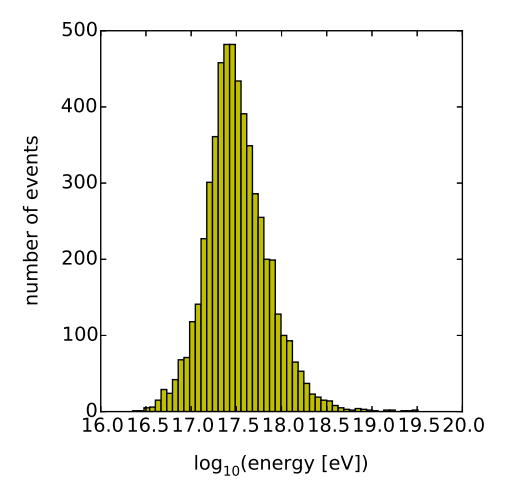

Figure 3: Energy distribution of AERA+SD hybrid data measured with the surface detector array.

reconstruction, the signals are corrected for the radio detector response and the time-dependent electric-field strength vector is calculated for further analyses. Shower parameters reconstructed with data from the other detector systems can be used as input during reconstruction.

Great efforts were made to calibrate the different AERA stations to determine absolute signal response and relative timing. All components of the analog signal processing chain were calibrated individually before deployment. For the antennas, detailed electromagnetic simulations were performed and various airborne calibration campaigns were carried out on site to calibrate the direction and frequency-dependent responses. The relative timing of the AERA stations is continuously monitored via their phase offset by measuring sine waves from a reference beacon [15]. The beacon is installed at the closest FD site and emits four precisely defined sine wave signals. Furthermore, signals from commercial aircrafts detected by the AERA stations are used to determine the timing. In combination, a precise timing calibration of about $2 \mathrm{~ns}$ is possible, whereas the station built-in GPS clocks exhibit drifts of tens of nanoseconds. Without precise timing information, analyses to determine the direction of the shower axis, interferometric approaches to enhance the detector sensitivity and analyses based on the radio wavefront would not be feasible.

\section{Data Set}

With AERA we have recorded a large radio data set of extensive air showers. Until the second of March 2015, more than 5500 air showers were detected by three or more antenna stations in coincidence with the surface detector array (AERA+SD hybrid events). The reconstructed arrival directions and the energy distribution as measured by the SD are shown in figures 2 and 3. During reconstruction a low level cut is made on a successfully reconstructed shower axis in agreement with the SD reconstruction within $20^{\circ}$. Most of the air showers are detected with 3 radio stations, but there are also air showers which are detected by more than 40 signal stations. During the stable operation phase of AERA24, about 50 air showers were recorded with three or more RDS per month which has increased to about 250 air showers per month for AERA124 after a short commissioning phase. In addition, 324 air showers were simultaneously detected with the fluorescence detector (AERA+SD+FD hybrid events). For these events, $X_{\max }$ is within the FD field of view and the measured profile allowed for an FD-based energy reconstruction. We also detected air showers in coincidence with the AMIGA muon counters. The data set contains 509 AERA+SD+AMIGA 

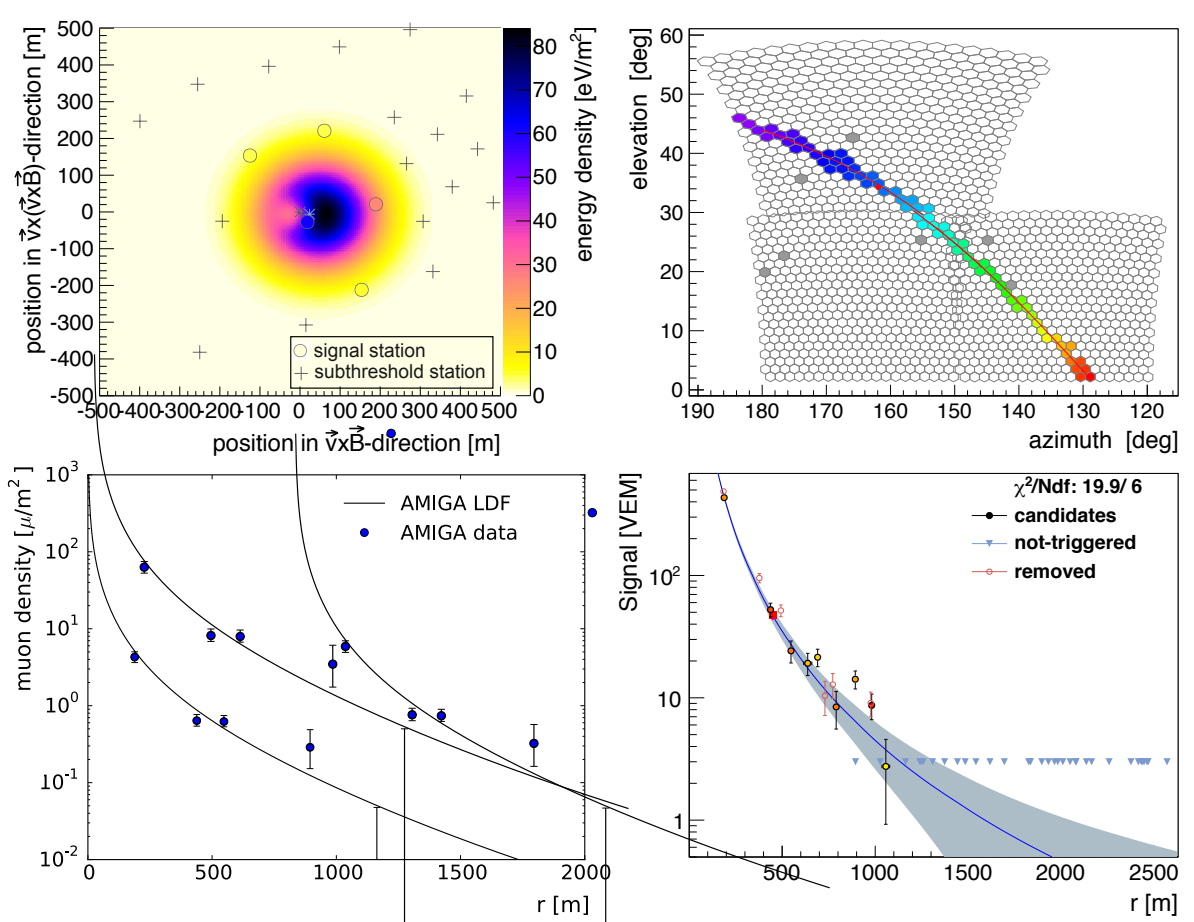

Figure 4: An extensive air shower detected with four different detector systems simultaneously. Top left: Radio footprint projected into the shower plane represented by the energy density measured with AERA124 and the fitted parametrization of the lateral distribution function (LDF) (background color). Top right: Longitudinal shower development measured with the fluorescence detector. Bottom left: Footprint of the air shower as measured with the muon counter of AMIGA. Bottom right: Particle signal in the surface detector stations including the classical one dimensional LDF fit.

hybrid events and 51 AERA+SD+FD+AMIGA hybrid events. An event display of a quadruple detection is shown in figure 4 . For about $5 \%$ of the data set, increased atmospheric electric fields have been measured. These data will be treated separately in upcoming analyses.

\section{Status and Prospects on Multi-Hybrid Analyses}

The three main properties of a cosmic ray are arrival direction, energy and particle type. AERA shows a sensitivity to all of these parameters which are discussed in the following.

The direction of the cosmic ray is taken from the axis of the air shower. This axis can be reconstructed by minimizing the difference between measured time at the RDS positions and the predicted time assuming a wavefront model and varying the shower geometry. A good first order approximation of the wavefront is a plane wave. Studies on the angular resolution of AERA are ongoing and only a comparison to the reconstructed axis from the SD can be given. The mean deviation between the two is $1.5^{\circ}$ and the distribution can be fitted by a Rayleigh function with $\sigma=0.45^{\circ}$.

The energy of the cosmic ray can be reconstructed from the radio signals by reconstructing the energy emitted in the measured radiation. Therefore, the electric field traces are converted into the energy density of the electromagnetic wave via the Poynting vector. The measured energy density at all positions of the signal stations is fitted with a two dimensional lateral distribution function [6]. 

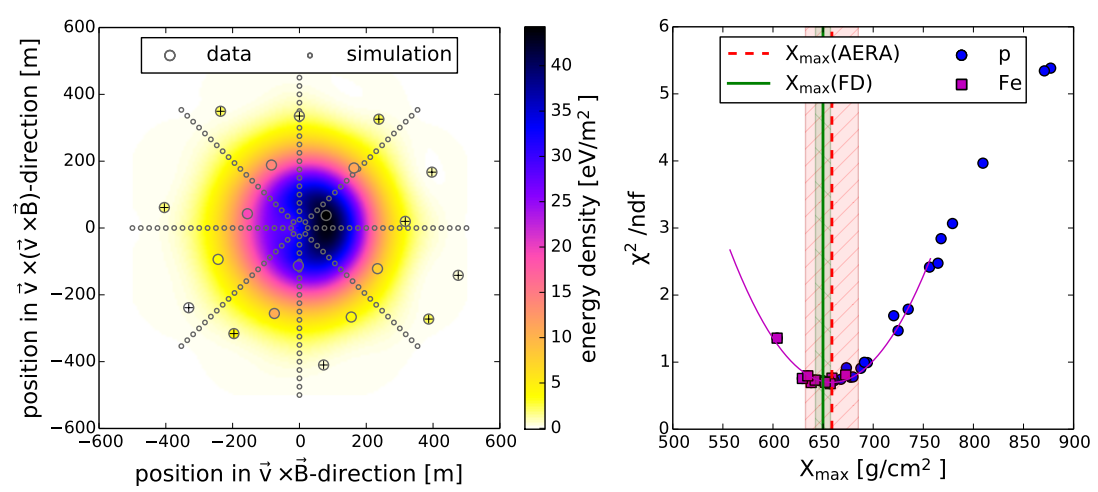

Figure 5: Reconstructed $X_{\max }$ value based on the fit quality of a set of CoREAS [16] simulations. Left: The two dimensional radio lateral distribution function. It displays the best fitting simulation (small circles) interpolated in the shower plane (background color map) as fitted to the energy densities measured by the AERA stations (colored circle), sub-threshold stations are additionally marked by a "+". Right: Distribution of reduced $\chi^{2}$ values for the $X_{\max }$ value of the individual simulations. The purple line is a parabolic fit to the distribution and the minimum is taken as the value of $X_{\max }$. The vertical lines indicate the reconstructed $\mathrm{X}_{\max }$ values from radio and FD together with the statistical uncertainties.

The spatial integral over this LDF gives the energy in 30-80 MHz radiation. The radiated energy is calibrated against the energy reconstructed by the SD. With this method, a resolution of $22 \%$ is obtained for a low station multiplicity ( $<5$ signal RDS) data set and $17 \%$ for a high station multiplicity set ( $>5$ signal RDS), both based on AERA24 measurements [2]. The radiation energy is subject of ongoing work as it can be predicted by Monte Carlo simulations without being strongly influenced by the uncertainties of the hadronic interaction models. Therefore the energy scale of cosmic rays can be studied based on classical electrodynamics. Four main studies targeting the reconstruction of $\mathrm{X}_{\max }$ are being carried out on the AERA+SD+FD hybrid data set. They are aiming at radio parameters and their calibration against the FD measurements or using those as comparison.

1. The width of the two dimensional LDF [7]. The further the emission region is away from the observer position, the larger the projection of the emission pattern gets.

2. The shape of the radio wavefront $[9,17]$. The precise appearance of the hyperbolic front is determined by the distance of the observer to the endpoint of the emission region. The closer this endpoint is to the observer, the more conical the wavefront gets.

3. The signal pulse shape [10]. Depending on the path length differences for radiation from early and late parts of the air shower, the pulses broaden if the emission region is closer to the observer. 4. The fourth study is targeting a combination of the parameters mentioned above by investigating the agreement between measurement and individual simulations of a set with various $X_{\max }$ values customized for the measured direction and energy. Therefore, the method used in [3] is modified to be applicable to the AERA+SD hybrid data. Figure 5 displays an air shower radio footprint and the best fitting simulation. The simulations are performed with CoREAS [16] using antenna positions oriented on the displayed star pattern. The output is interpolated and fitted to the data by varying the core position and the overall scaling. Simulations with different $X_{\max }$ values result in different $\chi^{2}$ values for the fit. The distribution of $\chi^{2}$ values as a function of the Monte Carlo $\mathrm{X}_{\max }$ values is fitted with a parabola and the minimum is taken as the value of the reconstructed $X_{\max }$. 

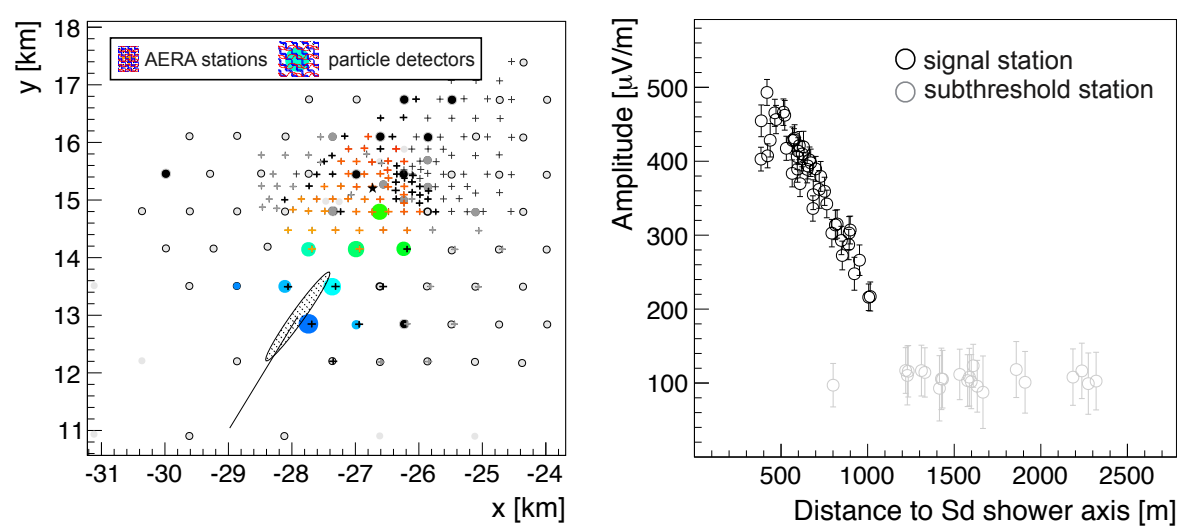

Figure 6: Event display for a horizontal shower measured with AERA153. Left: Array plot, where the crosses indicate radio antenna stations and the circles indicate surface detector stations. The size of the circle is scaled with the measured particle signal. The color indicates the signal timing. The reconstructed direction based on the surface detector data is indicated by the line together with the core position (ellipse). Right: LDF in terms of maximum amplitude as function of the distance to the air shower axis.

Another interesting target for hybrid analyses are horizontal air showers as complementary information can be obtained from radio and particle detectors. As horizontal air showers experience a larger column density of atmosphere before reaching the observer, the electromagnetic part of the shower is already absorbed and only the muonic component is detected by particle detectors. The radiation produced in the air shower is not absorbed however and can be detected. Therefore, the muonic and the electromagnetic parts of the shower can be measured independently by radio and particle detectors. Furthermore, horizontal air showers are interesting as they have large radio footprints due to the large distance to the radio source and projection effects on ground. Therefore, inclined air showers can still be detected with a rather sparse radio detector array allowing for large scale observatories and high statistics, even at the highest energies. The extension performed from AERA124 to AERA153 is dedicated to measuring horizontal showers. An event display of an air shower detected with AERA153 is shown in figure 6. 44 RDS have measured a significant signal for this air shower with an energy of $1.3 \times 10^{18} \mathrm{eV}$ and a zenith angle of $78^{\circ}$.

\section{Conclusions}

At the Pierre Auger Observatory radio emission from air showers is routinely measured simultaneously with other shower observables. With the data of AERA124 already recorded, high statistics radio data are now available in coincidence with the surface detector array. More than 300 air showers are also measured with the fluorescence detector. Combined, these data form the base for analyses targeting arrival direction, energy, and composition of the cosmic rays. The direction reconstruction is in good agreement with the surface detector reconstruction. We also developed a method for the energy reconstruction based on the measured energy density with a resolution of $17 \%$. Based on this method, the determination of the cosmic ray energy scale from first principles comes within reach. The mass determination is a target of intense research and various composition sensitive parameters have been identified. The experimental calibration of these parameters is carried out against fluorescence detector data. Horizontal air showers are targeted with the third stage of AERA and events with more than 50 signal stations have already been detected. 


\section{References}

[1] T. Huege, The Renaissance of Radio Detection of Cosmic Rays, Brazilian Journal of Physics 44 (2014) 520-529, [1310 . 6927].

[2] C. Glaser for the Pierre Auger Collaboration, The Energy Content of Extensive Air Showers in the Radio Frequency Range of 30-80 MHz, Proc. 34th ICRC, The Hague, The Netherlands (2015).

[3] LOFAR Collaboration, S. Buitink et al., Method for high precision reconstruction of air shower $X_{\max }$ using two-dimensional radio intensity profiles, Physical Review D 90 (2014), no. 8082003 , [1408.7001].

[4] LOPES Collaboration, S. Buitink et al., Amplified radio emission from cosmic ray air showers in thunderstorms, Astronomy \& Astrophysics 467 (2007) 385-394, [astro-ph/ 0702432 ].

[5] Pierre Auger Collaboration, A. Aab et al., Probing the radio emission from air showers with polarization measurements, Physical Review D 89 (2014), no. 5 052002, [1402 . 3677].

[6] A. Nelles et al., A parameterization for the radio emission of air showers as predicted by CoREAS simulations and applied to LOFAR measurements, Astroparticle Physics 60 (2015) 13-24, [1402.2872].

[7] LOFAR Collaboration, A. Nelles et al., The radio emission pattern of air showers as measured with LOFAR - a tool for the reconstruction of the energy and the shower maximum, JCAP 5 (2015) 18, [1411. 7868].

[8] LOFAR Collaboration, A. Corstanje et al., The shape of the radio wavefront of extensive air showers as measured with LOFAR, Astroparticle Physics 61 (2015) 22-31, [1404.3907].

[9] LOPES Collaboration, W. D. Apel et al., The wavefront of the radio signal emitted by cosmic ray air showers, JCAP 9 (2014) 25, [1404 .3283].

[10] S. Grebe for the Pierre Auger Collaboration, Spectral index analysis of the data from the Auger Engineering Radio Array, American Institute of Physics Conference Series 1535 (2013) 73-77.

[11] Pierre Auger Collaboration, A. Aab et al., The Pierre Auger Cosmic Ray Observatory, accepted for publication in Nucl. Instrum. Meth. A (2015) [1502.01323].

[12] Pierre Auger Collaboration, P. Abreu et al., Antennas for the detection of radio emission pulses from cosmic-ray induced air showers at the Pierre Auger Observatory, Journal of Instrumentation 7 (2012) $11 \mathrm{P},[1209.3840]$.

[13] D. Charrier for the CODALEMA Collaboration, Antenna development for astroparticle and radioastronomy experiments, Nucl. Instrum. Meth. A 662 (Jan., 2012) 142.

[14] Pierre Auger Collaboration, P. Abreu et al., Advanced functionality for radio analysis in the Offline software framework of the Pierre Auger Observatory, Nucl. Instrum. Meth. A 635 (2011) 92-102, [1101.4473].

[15] LOPES Collaboration, F. G. Schröder et al., New method for the time calibration of an interferometric radio antenna array, Nucl. Instrum. Meth. A 615 (2010) 277-284, [1 002 . 3775].

[16] T. Huege, M. Ludwig, and C. W. James, Simulating radio emission from air showers with CoREAS, American Institute of Physics Conference Series 1535 (2013) 128-132, [1301 . 2132].

[17] Q. Dorosti Hasankiadeh for the Pierre Auger Collaboration, Advanced Reconstruction Strategies for the Auger Engineering Radio Array, 6th Conference on Acoustic and Radio EeV Neutrino Detection, Annapolis, Maryland, USA (2014). 